\title{
Muscle degeneration in inguinal hernia specimens
}

\author{
G. Amato - A. Agrusa - G. Romano • \\ G. Salamone $\cdot$ G. Gulotta $\cdot$ F. Silvestri • \\ R. Bussani
}

Received: 15 June 2011/Accepted: 1 October 2011/Published online: 21 October 2011

(C) Springer-Verlag 2011

\begin{abstract}
Background There are few articles in the literature reporting the histological changes of groin structures affected by inguinal hernia. A deeper knowledge of this matter could represent an important step forward in the identification of the causes of hernia protrusion. This study aimed to recognise the pathological modifications of muscular structures in autopsy specimens excised from tissues surrounding the hernia orifice.

Methods Inguinal hernia was identified in 30 autopsied cadavers, which presented different varieties of hernia, including indirect, direct and mixed. Tissue specimens were resected for histological study from structures of the inguinal area surrounding the hernia opening, following a standardised procedure. The histological examination was focussed on the detection of structural changes in the muscle tissues. The results were compared with biopsy specimens resected from corresponding sites of the inguinal region in a control group of 15 fresh cadavers without hernia.

Results Significant modification of the muscular arrangement of the inguinal area was recognized. Pathological alterations such as atrophy, hyaline and fibrotic degeneration, as well as fatty dystrophy of the myocytes were detected. These findings were observed consistently
\end{abstract}

G. Amato $(\bowtie) \cdot$ A. Agrusa · G. Romano · G. Salamone ·

G. Gulotta

Department of General Surgery, Urgency, and Organ

Transplantation, University of Palermo, via del Vespro 129,

90100 Palermo, Italy

e-mail: amatomed@gmail.com

F. Silvestri · R. Bussani

Department of Pathological Anatomy and Histology,

University of Trieste, Strada di Fiume 447, 34149 Trieste, Italy in the context of multistructural damage also involving vessels and nerves. In cadavers with hernia these alterations were always present independent of hernia type. No comparable damage was found in control cadavers without hernia.

Conclusions The high degree of degenerative changes in the muscle fibres in the inguinal area involved in hernia protrusion described in this report seems to be consistent with chronic compressive damage. These alterations could embody one important factor among the multifactorial sources of hernia genesis. Conjectures concerning its impact on the physiology and biodynamics of the inguinal region are made. The relationship between the depicted degenerative injuries and the genesis of inguinal hernia is also a focus of discussion in this article.

Keywords Inguinal hernia - Etiology $\cdot$ Muscles · Fibrosis · Hyalin $\cdot$ Muscular dystrophy

\section{Introduction}

Despite constant progress in surgical techniques and materials, the genesis of inguinal hernia still represents an unanswered question [1-3]. A recent study summarising the state of the art in hernia topics clearly demonstrated that no clear advancement has been made concerning the dilemma of hernia genesis [4]. Even the latest theories based on collagen chain modifications and their relationship with inguinal hernia development do not seem to have disclosed a full link to hernia development [4-8]. Consequently, the surgical community is still searching for an effective and evidence-based pathogenetic model that demonstrates why a hernia arises in the inguinal area. However, the latest investigative attempts focused on the 
physiology and biodynamics of the inguinal region looks promising. Following these recent studies, important advances have been made. The demonstration of a "sphincter like" motion of the internal inguinal ring after its dilation [9], as well as the detection of histological modifications of the abdominal wall structures in patients with hernia [10-12], represents an additional incentive to intensify the investigation. The histological findings described in this report could further clarify how the inguinal area becomes weakened and is no longer able to fulfil its biomechanical role of providing dynamic protection for the groin, thus allowing the protrusion of an inguinal hernia.

\section{Materials and methods}

Inguinal hernias were identified in 30 autopsied cadavers. In these corpses, we detected 11 indirect inguinal hernia type 1 according to Nyhus; 8 indirect inguinal hernia type 2; 6 direct inguinal hernia type 3a; five other cadavers had direct inguinal hernia type $3 \mathrm{~b}$. The autopsies were carried out within $48 \mathrm{~h}$ of death. The mean age was 68 years (range 45-82 years). In order to achieve an appropriate control of the data to be compared between subjects with and without hernia, we developed a standardised procedure for the excision of the specimen from areas close to and surrounding the hernia opening. This allowed the excision of biopsy samples from tissue that corresponds to equivalent sites in cadavers both with or without hernia, even though there were different anatomical landmarks. In cadavers with hernia, we utilised the hernia opening as the key landmark (Fig. 1). Bisecting the hernia defect with a vertical line, we used the central point of the lower border as the initial point. From here, a line was drawn at $45^{\circ}$ to the right of vertical, out until the upper border of the defect was reached. Distances along this line of 0.5, 1.5 and $2.5 \mathrm{~cm}$ away from the border were measured and a full thickness biopsy of $0.5 \mathrm{~cm} \times 0.5 \mathrm{~cm}$ was taken at each point.

This procedure was repeated at $45^{\circ}$ to the left of the vertical, again with a line drawn along this angle, then measurements taken along this line above the hernia border of $0.5,1.5$ and $2.5 \mathrm{~cm}$, at which point biopsies were taken. This procedure was carried out in both direct and indirect hernias. To provide an appropriate control, biopsies were taken from the inguinal region of 15 male cadavers without hernia. The control autopsied bodies had a mean age of 65 years (range 48-79 years). To take control specimens from cadavers without hernia, two measurement sets were created. One as a control for direct hernia, and one as a control for indirect hernia. For indirect hernia, the exact procedure described above was imitated using the internal

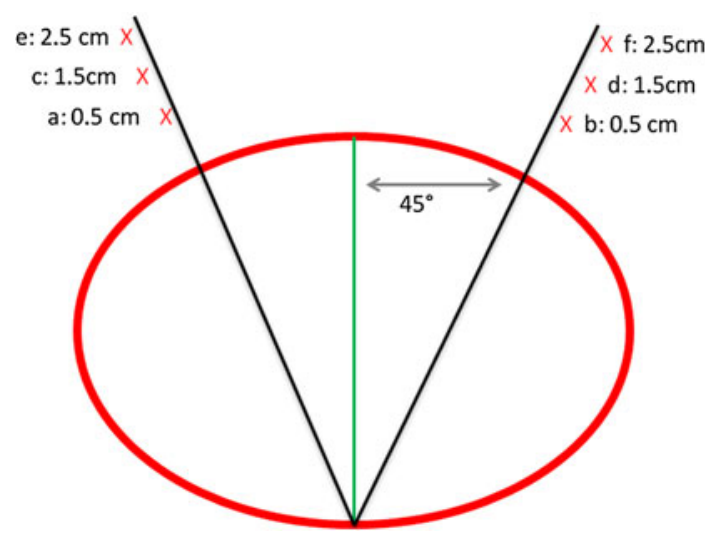

Fig. 1 Biopsy sites in subjects with inguinal hernia. Red ring internal inguinal ring in indirect inguinal hernia (or hernia opening in direct inguinal hernia), green line connects the lowest and the highest point of the hernia opening. Starting from the lowest point, a $45^{\circ}$ inclined black line transverses the figure to the right and on the left. Six excisions (X) were made: $a, b 0.5 \mathrm{~cm}$ above the junction of both $45^{\circ}$ angled lines; $c, d 1.5 \mathrm{~cm}$ above the junction of both $45^{\circ}$ angled lines; $e, f 2.5 \mathrm{~cm}$ above the junction of both $45^{\circ}$ angled lines

ring as the landmark instead of an actual indirect hernia. However, for direct hernia we had to modify the procedure to account for the hernia defects of the direct hernia being displaced from the inguinal ring. To achieve a control sample for direct hernia we used the inguinal ligament as the key landmark. Parallel to this ligament and above it we drew three lines at a distance of $0.5,1.5$ and $2.5 \mathrm{~cm}$. We then excised biopsy samples every $0.5 \mathrm{~cm}$ along these three lines, starting $0.5 \mathrm{~cm}$ medial to the epigastric vessels until $0.5 \mathrm{~cm}$ from the lateral border of the rectus muscle. In this way, we realized an acceptable histological mapping of the entire fossa inguinalis media. Although not a perfect solution, we felt that this repeatable measurement method of direct and indirect hernia, as well as the use of direct and indirect controls, gave us a significant comparison of tissue samples from the same anatomical areas in cadavers with hernia and controls without hernia. All tissue specimens were fixed in $10 \%$ neutral buffered formalin for at least $12 \mathrm{~h}$. After routine tissue processing, sections were cut at 4-6 mm and stained with Azan-Mallory, Weigert van Gieson, NSE, LCA and Hematoxylin eosine.

\section{Results}

The tissue specimens revealed several significant histological modifications concerning the structures bordering the hernia. Evidence of muscular atrophy was a distinctive characteristic in all tissue samples (Fig. 2). This was accompanied by hyaline and fibrotic degeneration of the muscle fibres, which were frequently surrounded by fatty 


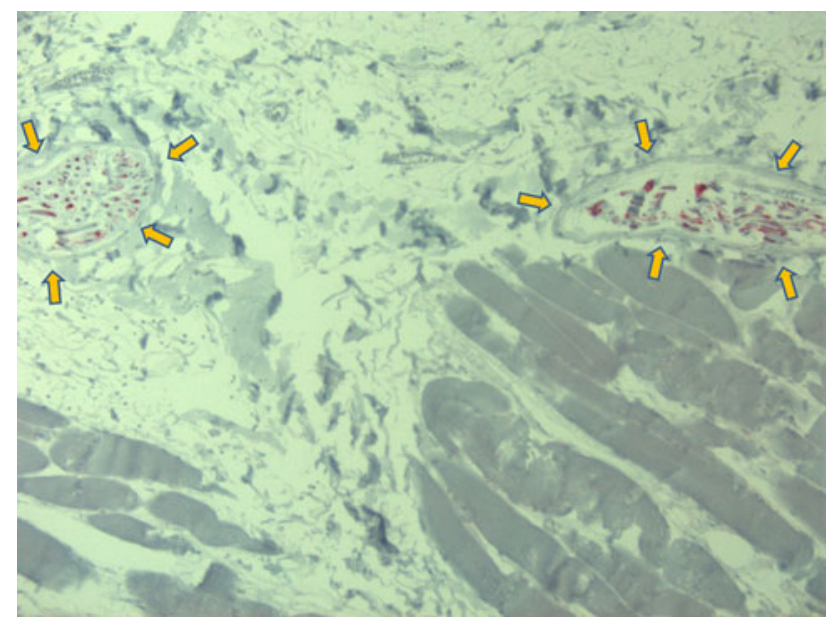

Fig. 2 Biopsy specimen excised $1.5 \mathrm{~cm}$ from indirect hernia border (Nyhus type 1): massive atrophy and fibrotic degeneration of the nerve axons (arrows) with thickening of the myelin sheath in a surround of fatty substitution (white areas) as well as atrophy and fibrosis of the muscle bundles (grey strips). NSE $\times 10$

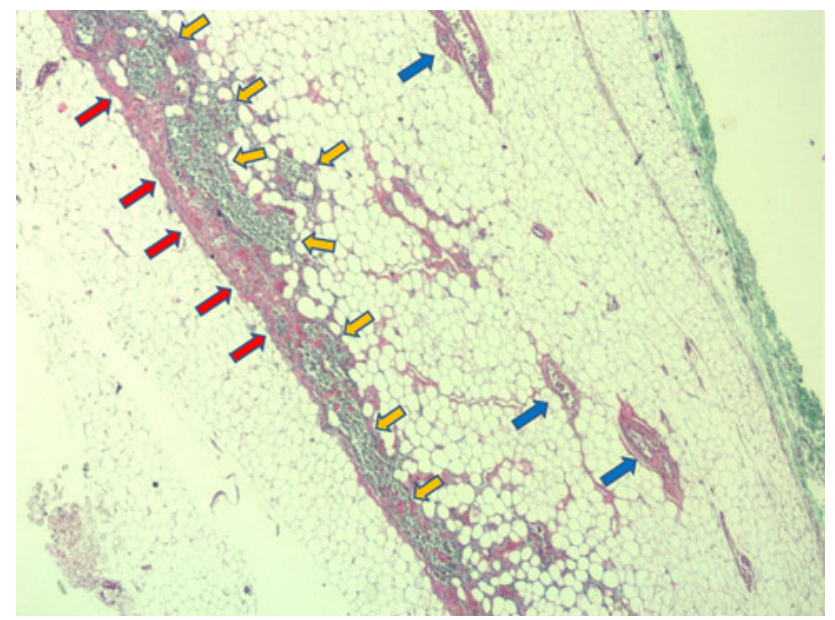

Fig. 3 Biopsy specimen excised $0.5 \mathrm{~cm}$ from indirect hernia border (Nyhus type 1). White spots Significant fatty dystrophy of the muscle fibers, red arrows residual fibrotic muscle bundle, yellow arrows lympho-histiocytic clusters, blue arrows venous congestion, green peritoneal sheath. Hematoxylin-eosin $\times 10$

dystrophy of the myocytes (Figs. 2, 3, 4, 5, 6, 7). Along with the muscular changes, the nervous structures clearly showed oedema, atrophy and fibrotic degeneration of the axons (Fig. 2). Inflammatory clusters with lympho-histiocytes and plasmacellular elements were consistently identified (Figs. 3, 6). Modifications of vascular structures were also observed frequently. Among these, vein fibrosis and venous congestion as well as arterial sub-occlusion-due to medial hypertrophy-and artery occlusion (Figs. 3, 4, 5). The described histological changes were detected regularly independently of the hernia type. On the other hand, no analogous histological damage was detected in the

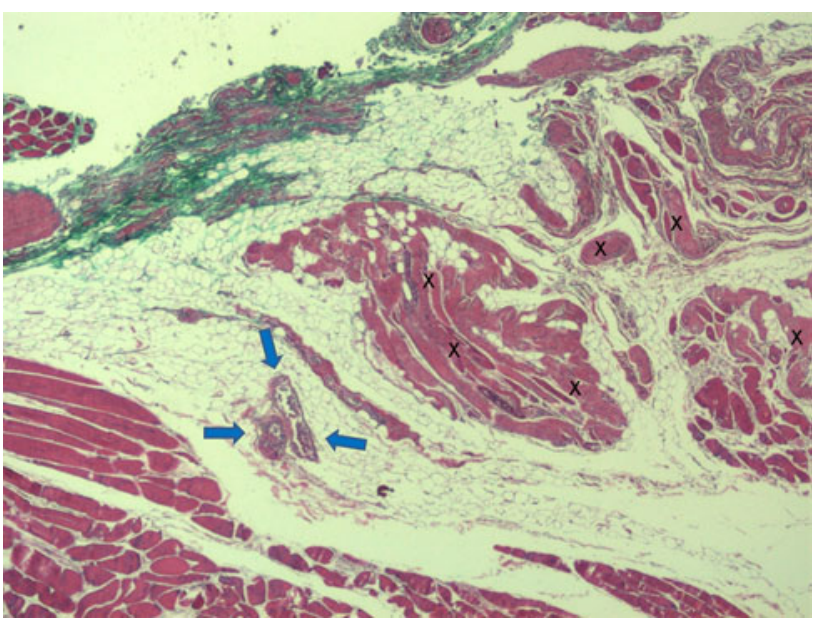

Fig. 4 Biopsy specimen excised $2.5 \mathrm{~cm}$ from direct hernia border (Nyhus type 3a). White spotty structures Fatty dystrophy, $X$ fibrosis of muscle fibers, blue arrows venous congestion, green peritoneal sheath-Hematoxylin-eosin $\times 10$

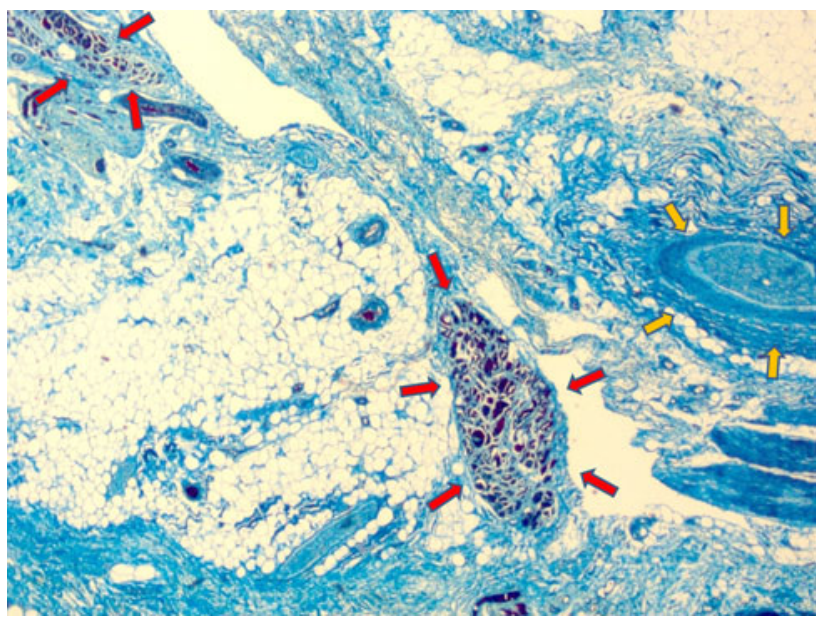

Fig. 5 Biopsy specimen excised $0.5 \mathrm{~cm}$ from indirect hernia border (Nyhus type 2). White spotty structures severe fibroadipous dystrophy with residual muscular bundles showing atrophy, red arrows fibrosis, yellow arrows artery with medial thickening and an endoluminal thrombus. Azan-Mallory $\times 2,5$

muscular structure of control samples. In the control group, the myocytes exhibited a normal structure with no noteworthy modifications. Only in a few middle-aged cadavers could a mild spotty fibrosis of the muscle fibers be detected. The presence of a negligible fibroadipous dystrophic altered area was seen in only one control specimen.

\section{Discussion}

In the scientific literature, among the many studies concerning ultrastuctural and biochemical modifications of 


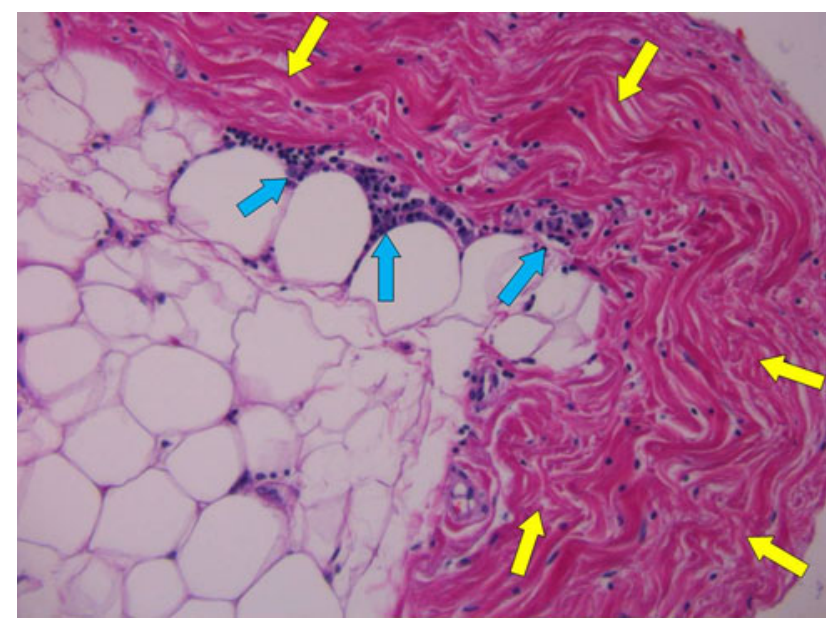

Fig. 6 Biopsy specimen excised $1.5 \mathrm{~cm}$ from mixed hernia border (Nyhus type 3b). Blue arrows Lymphoplasmacellular clusters in the transition zone between fatty muscle dystrophy (white circular elements), yellow arrows large hyaline fibrotic band. Hematoxylin$\operatorname{eosin} \times 20$

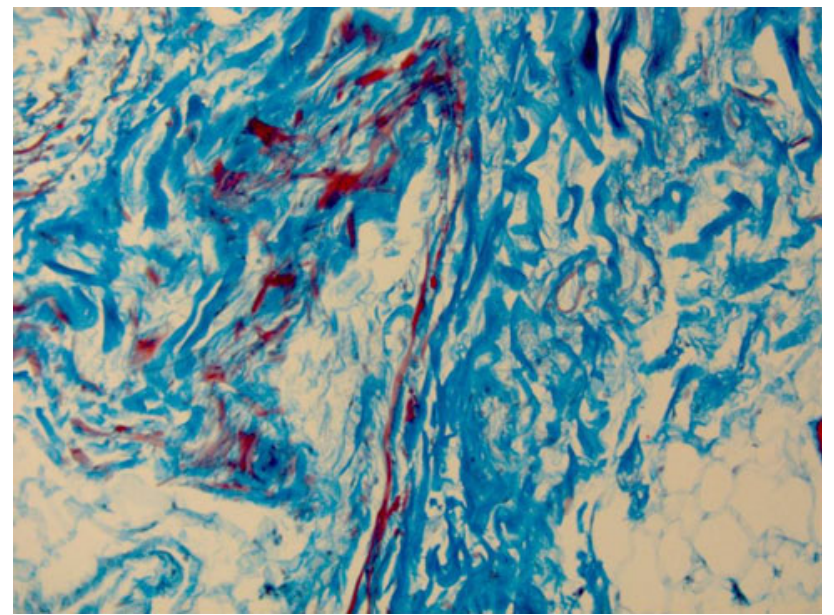

Fig. 7 Biopsy specimen excised $0.5 \mathrm{~cm}$ from direct hernia border (Nyhus type 3b). Blue Extreme fibrotic substitution of the muscle fibers, red residual atrophic myocytes. Azan-Mallory $\times 10$

inguinal structures [13-15], only a few scientific reports highlight the histological modifications in inguinal hernia protrusion. In our opinion, there has long been an unacceptable lack of scientific investigation concerning this topic. The missing histological evidence seems to represent important and missing knowledge on how structural modifications could impair the physiology of abdominal wall structures. Thus the real impact of these alterations in the kinetics of the groin still remains unknown. Only recently has a small series of scientific reports highlighted the histological features in inguinal structures affected by hernia protrusion [10-12]. In these latter reports, in addition to evidence of nervous and vascular changes as well as inflammatory infiltration, the presence of various degrees of muscular damage, such as atrophy, hyaline and fibrotic degeneration, as well as fatty dystrophy, has been evidenced as a collateral finding. The present study, which was aimed specifically at the detection of muscular damages in the herniated groin, fully confirms the abovementioned observations. In fact, atrophy of the muscle fibers as well as inflammatory clusters composed by lympho-histiocytic or plasmacellular elements, were observed within the excised tissue specimens. In addition, the complete scale of muscular tissue degeneration was witnessed in the biopsy samples: from the fibrotic to the hyaline, along with fatty dystrophy of the myocytes. Among these, the most important finding was the steady occurrence of a significant fatty dystrophy of the myocytes. The fibroadipous (fatty) dystrophy of muscle fibers was observed regularly between the fibrotic and the hyaline degeneration of the muscle bundles, which resulted in a clear distortion of the muscular geometry.

Fatty dystrophy is a well documented degeneration of muscle fibers due to chronic ischemia. It is described frequently in cardiac tissue samples as a result of myocardial infarction [16]. The pathway concerning this kind of pathological modification in muscle cells, from hypoxia to the fatty dystrophy, has been demonstrated biochemically and the results are well established $[16,17]$. Considering the impact these phenomena could have on the muscular structure of the inguinal area, it could be envisaged that ischemic damage might well be a consequence of the constant orthostatic compression exerted by the abdominal viscera in this region. Indeed, changes in muscular structure due to long term compression have been confirmed in the literature [18]. Nevertheless, the histological changes in muscular structure detected are consistent, and could hint at an explanation as to why this kind of myotendinous disbanding leads to weakness of the inguinal region and thus promotes the protrusion of a hernia. Other, apparently minor, histological changes, such as inflammatory infiltrate, vascular alterations and changes in nerve endings, should be considered as specific modifications in the framework of a multifactorial disease, which, over time, reduces the physical barrier of the inguinal region. The lympho-histocytic and plasmacellular infiltration could be seen as an inflammatory response to the compression, leading to additional stress on myocytes; this occurrence has also been reported in the literature [19].

The structural modification of veins (congestion and fibrosis), as well as the reduction in the artery due to thickening of the media, seems to be a consequence of long-term tissue compression. Of particular significance could be the alterations of the nerve axons witnessed in the excised tissues, where edema, fibrosis and atrophy were clearly seen. In addition, all the damaged nerve endings showed thickening of the myelinic sheath. This, as 
described in the literature, corresponds specifically to a compressive nerve damage $[11,20,21]$. As a consequence, additional muscle damage (other than direct compression) can be imagined: muscle atrophy following a gradual worsening, or even total blockade of the motor nervous impulse to the muscle bundles. Muscle atrophy leads to loss of contractile force and loss of muscle mass-reducing wall thickness. All these combined events could explain why the abdominal wall in the inguinal area weakens, thus leading to hernia protrusion. The reported tissue changes found in specimens excised from areas close to inguinal hernias in fresh cadavers seem to enforce the hypothesis of a multifactorial damage leading to a significant weakening of the abdominal wall in the inguinal area. The described modifications of specific structures, such as muscle degeneration, involved in hernia protrusion are proposed as a supplementary contributing factor in understanding the causes of hernia genesis. In our opinion, furthering the study of modifications of the tissue structures bordering the hernia opening could provide the surgical community with additional opportunities to clarify the dilemma of hernia genesis. The disclosure of all pathogenic aspects of hernia disease could lead to more physiological hernia repair techniques by means of a new generation of implants that are better tailored to the physiology and biodynamics of the groin.

\section{References}

1. Lytle WJ (1961) Anatomy and function in hernia repair. Proc R Soc Med 54:967-970

2. Stoppa R (1984-2004) Como se forma una hernia inguinal? Actualizacion en hirugia del aparato digestivo. Fundacion MMA 8:469-473

3. Flament JB (2006) Funktionelle Anatomie der Bauchwand. Chirurg 77:401-407

4. Read RC (2003) Recent advances in the repair of groin herniation. Curr Probl Surg 40(1):13-79

5. Peacock EE, Madden JW (1974) Studies on the biology and treatment of recurrent inguinal hernia II. Morphological changes. Ann Surg 179:567-571
6. Klinge U, Binnebösel M, Mertens PR (2006) Are collagens the culprits in the development of incisional and inguinal hernia disease? Hernia 10(6):472-477

7. Pans A (2002) Biochemical study of collagen in adult groin hernias. J Surg Res 95(2):107-113

8. Friedman DW, Friedman DW, Boyd CD, Norton P, Greco RS, Boyarsky AH, Mackenzie JW, Deak SB (1993) Increases in type III collagen gene expression and protein synthesis in patients with inguinal hernias. Ann Surg 218(6):754-760

9. Amato G, Sciacchitano T, Bell SG, Romano G, Cocchiara G, Lo Monte AI, Romano M (2009) Sphincter-like motion following mechanical dilation of the internal inguinal ring during indirect inguinal hernia procedure. Hernia 13:67-72

10. Amato G, Marasa L, Sciacchitano T, Bell SG, Romano G, Gioviale MC, Lo Monte AI, Romano M (2009) Histological findings of the internal inguinal ring in patients having indirect inguinal hernia. Hernia 13:259-262

11. Amato G, Ober E, Romano G, Salamone G, Agrusa A, Gulotta G, Bussani R (2011) Nerve degeneration in inguinal hernia specimens. Hernia 15:53-58

12. Amato G, Romano G, Salamone G, Agrusa A, Saladino VA, Silvestri F, Bussani R (2011) Damage to the vascular structures in inguinal hernia specimens. Hernia. doi:10.1007/s10029-01 $1-0847-4$

13. Klinge U, Binnebösel M, Mertens PR (2006) Are collagens the culprits in the development of incisional and inguinal hernia disease? Hernia 10(6):472-477

14. Pans A (2002) Biochemical study of collagen in adult groin hernias. J Surg Res 95(2):107-113

15. Friedman DW, Boyd CD, Norton P, Greco RS, Boyarsky AH, Mackenzie JW, Deak SB (1993) Increases in type III collagen gene expression and protein synthesis in patients with inguinal hernias. Ann Surg 218(6):754-760

16. Silvestri F, Bussani R (1990) Hypoxic right ventricular cardiomyopathy. A morphological and pathogenetic study on the myocardial atrophy and fatty infiltration. Pathologica 82:593-616

17. Basso C, Thiene G, Corrado D, Angelini A, Nava A, Valente M (1996) Arrhythmogenic right ventricular cardiomyopathy. Dysplasia, dystrophy, or myocarditis? Circulation 94(5):983-991

18. Bai YH, Takemitsu M, Atsuta Y, Takemitsu Y (1998) Pathology study of rabbit calf muscles after repeated compression. J Orthop Sci 3(4):209-215

19. Shoen FJ (2005) Blood vessels. In: Kumar V, Abbas AK, Fausto N (eds) Robbins and Cotran pathologic basis of disease. Saunders, Philadelphia, pp 513-515

20. Kincaid JC, Stewart JD (1999) Focal peripheral neuropathies. J Clin Neuromuscul Dis 1(2):113

21. Penkert G (1998) Nerve compression syndrome-1. Chirurg 69:1114-1122 